\title{
Intraindividual Comparison between the Contrast-Enhanced Golden-Angle Radial Sparse Parallel Sequence and the Conventional Fat-Suppressed Contrast-Enhanced T1- Weighted Spin-Echo Sequence for Head and Neck MRI
}

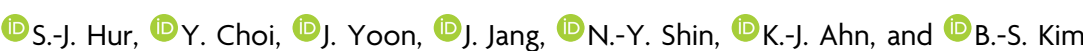

\begin{abstract}
BACKGROUND AND PURPOSE: The golden-angle radial sparse parallel-volumetric interpolated breath-hold (GRASP-VIBE) sequence is a recently introduced imaging technique with high resolution. This study compared the image quality between conventional fatsuppressed T1-weighted TSE and GRASP-VIBE after gadolinium enhancement in the head and neck region.
\end{abstract}

MATERIALS AND METHODS: Data from 65 patients with clinical indications for head and neck MR imaging between September 2020 and January 2021 were retrospectively reviewed. Two radiologists assessed the overall image quality, overall artifacts, and image conspicuities in the oropharynx, hypopharynx, and cervical lymph nodes according to 5-point scores (best score: 5). Interobserver agreement was assessed using weighted $\kappa$ statistics. The SNR and contrast-to-noise ratio were calculated and compared between the 2 sequences using a paired Wilcoxon signed rank test.

RESULTS: The analysis included 52 patients (mean age, 60 [SD, 14] years; male, 71.2\% [37/52]) who were mostly diagnosed with head and neck malignancies (94.3\% [50/52]). $\kappa$ statistics ranged from slight agreement in cervical lymph node conspicuity $(\kappa=0.18)$ to substantial agreement in oropharyngeal mucosal conspicuity $(\kappa=0.80)(\kappa$ range, 0.18-0.80). Moreover, GRASP-VIBE demonstrated significantly higher mean scores in overall image quality (4.68 [SD, 0.41] versus 3.66 [SD, 0.73]), artifacts (4.47 [SD, 0.48] versus 3.58 [SD, 0.71]), oropharyngeal mucosal conspicuity (4.85 [SD, 0.41] versus 4.11 [SD, 0.79]), hypopharyngeal mucosal conspicuity (4.84 [SD, 0.34 ] versus 3.58 [SD, 0.81]), and cervical lymph node conspicuity (4.79 [SD, 0.32] versus 4.08 [SD, 0.64]) than fat-suppressed T7weighted TSE (all, $P<.001$ ). Furthermore, GRASP-VIBE demonstrated a higher SNR (22.8 [SD, 11.5] versus 11.3 [SD, 5.6], $P<.001)$ and contrast-to-noise ratio (4.7 [SD, 5.4] versus 2.3 [SD, 2.7], $P=.059)$ than fat-suppressed T1-weighted TSE.

CONCLUSIONS: GRASP-VIBE provided better image quality with fewer artifacts than conventional fat-suppressed T1-weighted TSE for the head and neck regions.

ABBREVIATIONS: CNR = contrast-to-noise ratio; GRASP-VIBE = golden-angle radial sparse parallel-volumetric interpolated breath-hold; T1-TSE $=$ fatsuppressed T1-weighted TSE

M

$\mathrm{R}$ imaging is an integral imaging technique for detailed anatomic assessment of complex head and neck regions. In particular, conventional gadolinium-enhanced fat-suppressed T1-weighted TSE (T1-TSE) is widely used in routine clinical

Received April 1, 2021; accepted after revision July 25.

From the Department of Radiology, Seoul St. Mary's Hospital, College of Medicine, The Catholic University of Korea, Seoul, Korea.

This research was supported by the Basic Science Research Program through the National Research Foundation of Korea funded by the Ministry of Education (2021RIIIA1A01040285).

Please address correspondence to Yangsean Choi, MD, Department of Radiology, Seoul St. Mary's Hospital, The Catholic University of Korea, College of Medicine, Seoul, Korea, 222 Banpo-daero, Seocho-gu, Seoul (06591), Korea; e-mail: phillipchoi007@gmail.com

- Indicates open access to non-subscribers at www.ajnr.org

http://dx.doi.org/10.3174/ajnr.A7285 settings. ${ }^{1-3}$ However, the image quality of T1-TSE in the lower neck region is often degraded by artifacts caused by pulsation, respiratory motion, and wide magnetic susceptibility variations. ${ }^{4-6}$ Therefore, conventional T1-TSE has its limitations when considering the need for detailed assessment of small, complex structures in the head and neck region.

The golden-angle radial sparse parallel-volumetric interpolated breath-hold (GRASP-VIBE) sequence is a recently introduced imaging technique that combines radial 3D T1-weighted spoiled gradient-echo (VIBE, Siemens; THRIVE, Philips Healthcare; LAVA, GE Healthcare), parallel imaging, and compressed sensing reconstruction for dynamic contrast-enhanced MR imaging. GRASP-VIBE continuously acquires radial spokes throughout the scan with contrast agent injection. Then, multiple phases of dynamic 3D T1-weighted images are reconstructed from highly 
Table 1: Detailed acquisition parameters of the 2 sequences

\begin{tabular}{lcc}
\hline \multicolumn{1}{c}{ Sequence } & GRASP-VIBE & T1-TSE \\
\hline FOV & $200 \times 200$ & $200 \times 200$ \\
Matrix & $224 \times 224$ & $384 \times 230$ \\
Section thickness (mm) & 4 & 4 \\
Gap (mm) & 0 & 1 \\
No. of slices & 26 & 32 \\
In-plane resolution (mm) & $1.04 \times 1.04$ & $0.52 \times 0.52$ \\
Echo-train & 1 & 4 \\
Flip angle & $12^{\circ}$ & $160^{\circ}$ \\
TR/TE (ms) & $4.1 / 1.9$ & $690 / 12$ \\
Bandwidth (Hz/px) & $496 \mathrm{~Hz} / \mathrm{px}$ & $310 \mathrm{~Hz} / \mathrm{px}$ \\
No. of excitations & 1 & 1 \\
Fat-suppression technique & Chemical shift & Dixon \\
& selective & \\
Scanning type & Gradient-echo & TSE \\
Scan time (min/sec) & $4 / 46$ & $2 / 6$ \\
No. of dynamic acquisitions & $95(2.5)$ & $\mathrm{NA}$ \\
\multicolumn{1}{c}{ (temporal resolution) (sec) } & $3 \mathrm{D}$ & $2 \mathrm{D}$ \\
Acquisition type & &
\end{tabular}

Note:-NA indicates not applicable. Consecutive patients who underwent head and neck
MRI between September 2020 and January 2021 $(n=65)$

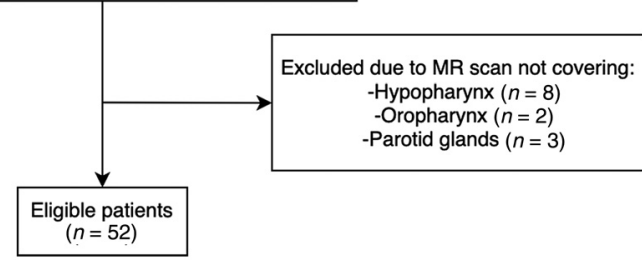

FIG 1. Flow chart of the patient-selection process.

undersampled radial spokes using iterative reconstruction. Because the continuous golden-angle sampling conserves the sampling uniformity of the $k$-space at any time point, any number of successive radial spokes are combined to make dynamic images, enabling various temporal resolutions and time points. Furthermore, GRASPVIBE gives a static 3D image after combination of all the radial spokes, which can be used as an enhanced 3D T1-weighted image. Also, because GRASP uses a radial trajectory where the center of the $k$-space is sampled every TR, it is more motion-robust at high spatial and temporal resolutions. ${ }^{7}$ Excellent GRASP-derived images have already been demonstrated with fewer motion and pulsation artifacts in prior liver, ${ }^{8}$ prostate, ${ }^{9}$ and breast ${ }^{10}$ studies.

A similar study by $\mathrm{Wu}$ et $\mathrm{al}^{11}$ applied a radial-volumetric interpolated breath-hold examination (VIBE) in the head and neck and found that radial VIBE was a viable motion-robust improvement over the conventional fat-suppressed T1-TSE. ${ }^{11}$ Our study attempted to apply a more novel GRASP-VIBE technique in the head and neck with a larger sample size and variable clinical indications, including preoperative staging and postoperative assessment, thereby reflecting a more comprehensive clinical setting.

We hypothesized that GRASP-VIBE would yield better images of the head and neck than conventional T1-TSE and that
Table 2: Baseline characteristics of included patients ${ }^{a}$

\begin{tabular}{lc}
\multicolumn{1}{c}{ Variable } & $N=52$ \\
\hline Age (mean) (yr) & $60(\mathrm{SD}, 14)$ \\
Sex & \\
Male & $37(71.2)$ \\
Female & $15(28.8)$ \\
Diagnosis & \\
Head and neck malignancies & $50(94.3)$ \\
Buccal & $1(1.9)$ \\
Floor of mouth & $1(1.9)$ \\
Vocal cord & $10(19.2)$ \\
Supraglottis & $1(1.9)$ \\
Hypopharynx & $7(13.5)$ \\
Salivary gland & $6(11.5)$ \\
Tongue & $13(25.0)$ \\
Tonsil & $10(19.2)$ \\
Other malignancies & $3(5.7)$ \\
Primary lesion visible & \\
Yes & $10(19.2)$ \\
No & $42(80.8)$ \\
Postoperative state & \\
Yes & $45(86.5)$ \\
No & $7(13.5)$ \\
\hline
\end{tabular}

${ }^{a}$ All values are presented as No. (\%) unless otherwise specified.

GRASP-VIBE would be a promising alternative imaging technique to T1-TSE. Therefore, the purpose of the current study was to qualitatively and quantitatively compare the image qualities of GRASP-VIBE and conventional T1-TSE sequences.

\section{MATERIALS AND METHODS Patients}

This single-center retrospective cohort study was approved by our institutional review board, and the requirement for informed consent was waived. Between September 2020 and January 2021, data from 65 consecutive patients who had undergone head and neck MR imaging for various clinical indications were reviewed. The inclusion criteria were as follows: 1) availability of simultaneously acquired gadolinium-enhanced GRASP-VIBE and T1-TSE sequences; 2) MR imaging scan area covering the nasopharynx to the subglottic region; and 3) visible left parotid glands and masseter muscles.

\section{GRASP-VIBE Acquisition}

GRASP-VIBE sequence data were acquired using a fat-saturated T1-weighted radial 3D gradient recalled-echo sequence. ${ }^{7}$ GRASPVIBE samples were characterized by a $k$-space with a stack-of-stars scheme, in which radial spokes are stacked along the partition direction. An angle increase of $111.25^{\circ}$ in consecutive spokes yields approximately uniform $k$-space coverage during acquisition. ${ }^{12}$ Moreover, compressed-sensing reconstruction was applied to minimize streaking artifacts caused by data undersampling in the radial acquisition. For this study, a 2.5-second temporal resolution (ie, 21 spokes/frame and a static image of 2427 radial views) was used.

\section{MR Imaging Acquisition}

All MR imaging examinations were performed using 3T scanners with 64-channel head and neck coils (Magnetom Vida; Siemens). Gadoteridol (ProHance; Bracco Diagnostics) had been intravenously administered at a rate of $1-2 \mathrm{~mL} / \mathrm{s} \quad(0.01 \mathrm{mmol} / \mathrm{kg}$ of 


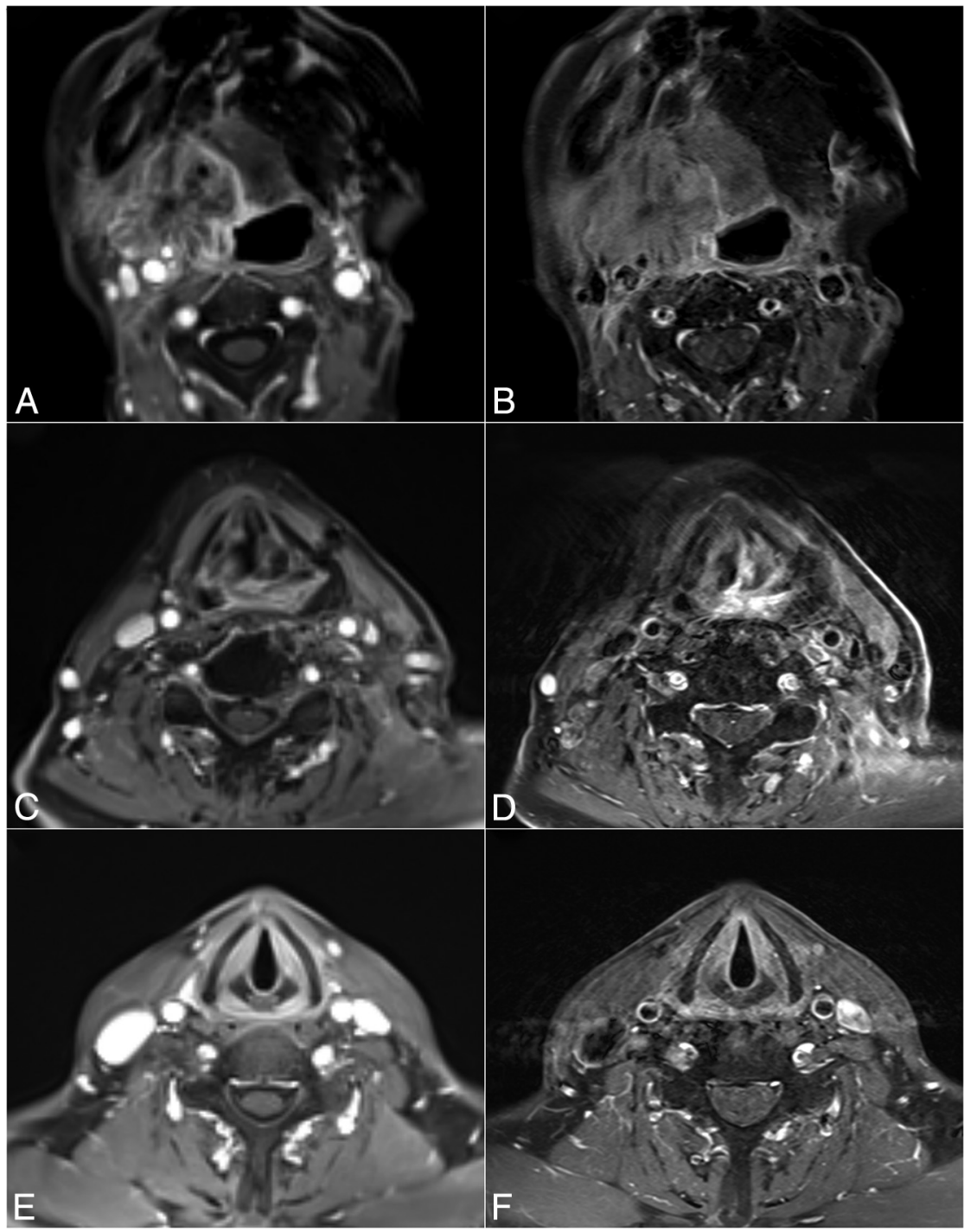

FIG 2. A 55-year-old female patient with a history of recurrent squamous cell carcinoma of the tongue (top row); the lesion is better delineated in GRASP-VIBE (A) than in TI-TSE (B). A 76-yearold male patient with a history of left hypopharyngeal cancer with transoral robotic surgery (middle row). Compared with GRASP-VIBE (C), a significant respiratory motion artifact is observed in T7-TSE (D). A 70-year-old male patient with a history of left vocal cord squamous cell carcinoma in situ and laser cordectomy (bottom row). Compared with GRASP-VIBE (E), there is a noticeable pulsation artifact from blood vessels in TT-TSE (F).

patient's clinical information during the review. The qualitative assessment included overall image quality, overall artifacts, and image conspicuities of the oropharyngeal, hypopharyngeal, and cervical lymphatic channels. All assessments were graded using a 5-point scoring system. For assessment of overall image quality and overall artifacts, scores were graded as $5=$ best, $4=$ good, $3=$ adequate, $2=$ poor, and $1=$ nondiagnostic. The artifacts under evaluation included respiratory motion artifacts, pulsation artifacts of nearby blood vessels, and susceptibility artifacts in the oral cavity. For the assessment of mucosa conspicuities, scores were graded as $5=$ sharp margin, $4=$ minimal blurring, $3=$ moderate blurring, $2=$ substantial blurring, and $1=$ nondiagnostic due to severe blurring.

\section{Quantitative Image Analysis}

For quantitative analysis, the first rater (R1) drew circular ROIs with areas ranging from 10 to $30 \mathrm{~mm}^{2}$ on the left parotid glands and masseter muscles. All ROIs were then reviewed by the second rater (R2). Image analyses were performed using an in-house PACS at a designated workstation. From the ROIs, the SNR and contrast-to-noise ratio $(\mathrm{CNR})$ were calculated using the following formulas: ${ }^{13-15}$

$$
S N R=\frac{S I_{\text {Parotid gland }}}{S D_{\text {Masseter muscle }}},
$$

$C N R=\frac{\left(S I_{\text {Parotid gland }}-S I_{\text {Masseter muscle }}\right)}{S D_{\text {Masseter muscle }}}$.

$\mathrm{SI}_{\text {Parotid gland }}$ is the mean signal intensity of the parotid glands, and

body weight). Approximately 5 seconds after gadoteridol administration, GRASP-VIBE was acquired. T1-TSE was acquired approximately 4-5 minutes after gadoteridol administration. The acquisition times for the GRASP-VIBE and T1-TSE were 4 minutes 46 seconds and 2 minutes 6 seconds, respectively. The details of the GRASP-VIBE and T1-TSE acquisition parameters are summarized in Table 1. All images were acquired under freebreathing conditions.

\section{Qualitative Image Analysis}

Two radiologist raters (raters 1 and 2 with 2 and 8 years of experience in head and neck radiology, respectively) independently reviewed the MR images. The raters were blinded to the

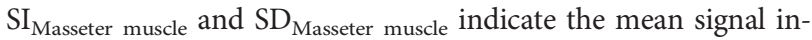
tensity and SD of the masseter muscles, respectively. All signal intensities and SDs were calculated within the ROIs.

\section{Statistical Analyses}

Interobserver agreement in the image-quality assessment for each category was assessed using weighted $\kappa$ coefficients. The imageassessment scores as well as the SNR and CNR between GRASPVIBE and T1-TSE were compared using a paired Wilcoxon rank sum test. Statistical significance was 2-sided and was set at $P<.05$. All statistical analyses were performed with $\mathrm{R}$ statistical and computing software, Version 4.10.1 (http://www.r-project. $\operatorname{org} /)$. 


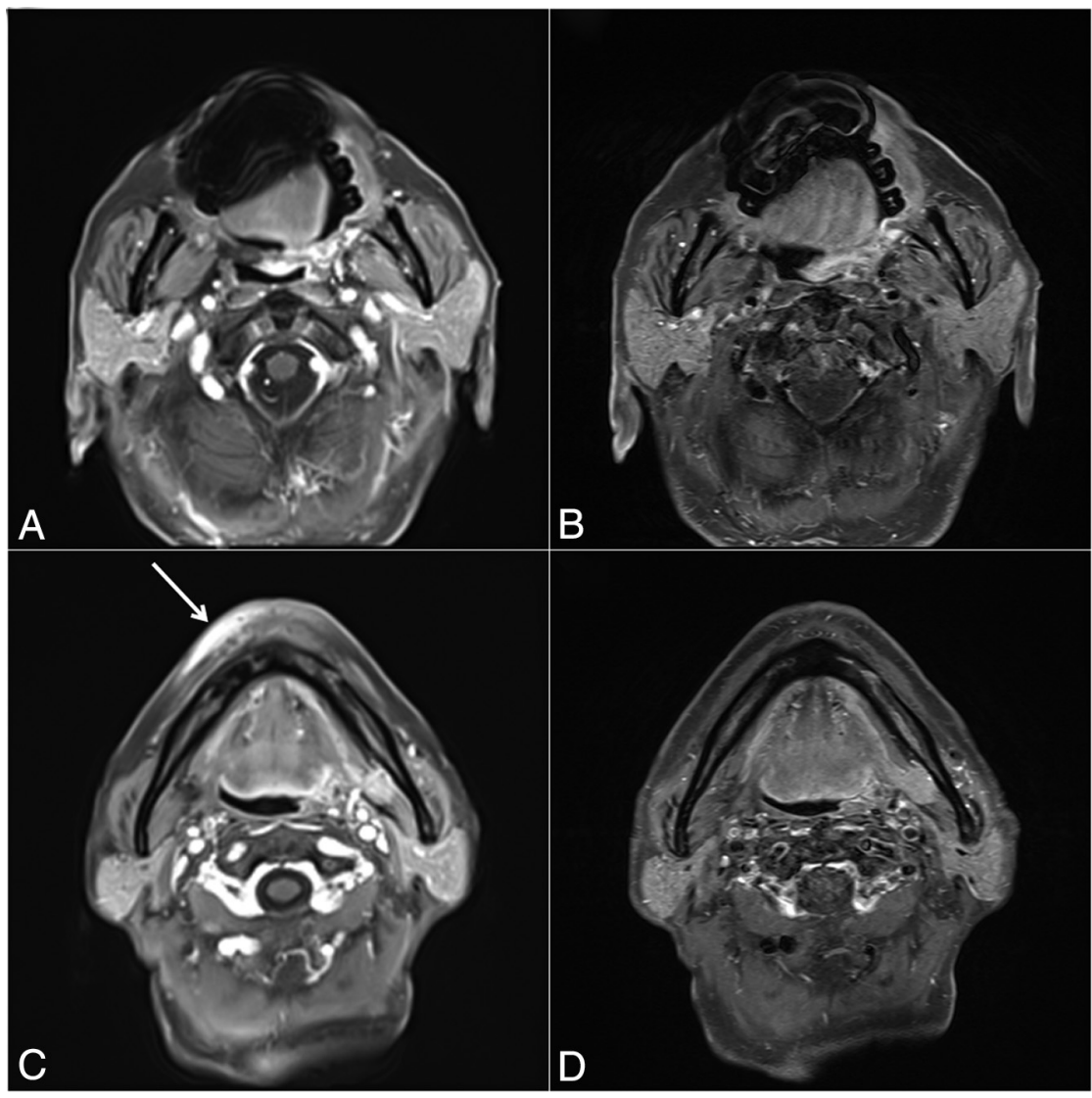

FIG 3. A 73-year-old male patient with a history of oropharyngeal squamous cell carcinoma of the right tonsil; the susceptibility artifacts due to dental amalgam are more prominent in GRASPVIBE $(A)$ than in TT-TSE (B). In right submental region of the same patient, fat suppression is weaker in GRASP-VIBE $(C)$ than in TT-TSE (D).

\section{RESULTS}

\section{Clinical Characteristics of Patients}

Among the initial 65 patients, 13 patients were excluded because their scans did not cover the hypopharynx $(n=8)$, oropharynx $(n=2)$, and parotid glands and masseter muscles $(n=3)$. After exclusion, 52 eligible patients remained for analysis (Fig 1). The baseline characteristics of eligible patients are summarized in Table 2. Among the 52 patients (mean age, 60 [SD, 14] years; 37 men and 15 women), 50 had head and neck malignancies, including tongue cancer $(13,25 \%)$, vocal cord cancer (10, 19.2\%), supraglottic cancer $(1,1.9 \%)$, tonsil cancer (10, 19.2\%), hypopharynx cancer $(7,13.5 \%)$, salivary gland cancer $(6,11.5 \%)$, buccal cancer $(1,1.9 \%)$, and floor of mouth cancer $(1,1.9 \%)$; and 3 had other malignancies (5.7\%). Forty-five patients (86.5\%) were examined during postoperative follow-up, and malignant lesions were visible in 10 patients (19.2\%). Representative MR imaging comparing the 2 sequences is illustrated in Figs 2 and 3.

\section{Qualitative Assessment}

The results of the qualitative assessment of the 2 sequences are summarized in Table 3 and graphically depicted in Fig 4 . The mean quality scores were significantly higher in GRASP-VIBE than in T1-TSE in all assessments, including overall image quality, overall artifacts, and image conspicuities of the oropharyngeal, hypopharyngeal, and cervical lymph nodes (all, $P<.001$ ).

Interobserver agreement was variable, as measured by $\kappa$ statistics; the agreement was substantial for the assessment of oropharyngeal mucosa conspicuity $(\kappa=0.80$, for GRASPVIBE; $\kappa=0.5$, for T1-TSE), whereas it was slight-to-moderate for the assessment of cervical lymph node conspicuity $(\kappa=0.18$ for GRASP-VIBE; $\kappa=$ 0.34 for T1-TSE).

\section{Quantitative Assessment}

The boxplots of the SNR and CNR of GRASP-VIBE and T1-TSE are depicted in Fig 5. The mean SNR of GRASP-VIBE (22.8 [SD, 11.5]) was significantly higher than that of T1TSE $(11.3$ [SD, 5.6]) $(P<.001)$. The mean CNR of GRASP-VIBE (4.7 [SD, 5.4]) was also higher than that of T1TSE (2.3 [SD, 2.7]), but without statistical significance $(P=.059)$.

\section{DISCUSSION}

In the current study, overall image quality, overall artifacts, and image conspicuities at different anatomic locations of the head and neck were compared between GRASP-VIBE and T1-TSE. Furthermore, the SNR and CNR of both sequences were quantitatively compared. GRASP-VIBE provided significantly better image quality and a higher SNR compared with the conventional T1-TSE sequence. Therefore, GRASP-VIBE could be a superior alternative to conventional T1-TSE for the assessment of the head and neck region.

Compared with T1-TSE, GRASP-VIBE showed higher scores in all qualitative assessments, including overall image quality, overall artifacts, and image conspicuities of the oropharyngeal, hypopharyngeal, and cervical lymphatic channels. GRASP-VIBE showed significantly higher scores, especially in terms of artifacts and hypopharyngeal mucosa conspicuity. This finding is due to pulsation and respiratory motion artifacts inherently associated with the T1-TSE sequence, which mainly affect the lower neck region and, in turn, degrade the image quality of the hypopharyngeal region.

In this regard, the findings of our study are clinically relevant for imaging assessment of hypopharyngeal and laryngeal head and neck cancers, whose MR imaging scans are often critically affected by pulsation artifacts from adjacent carotid arteries and jugular veins as well as respiratory motion artifacts from involuntary movement due to free breathing or swallowing. Minimizing such artifacts would be beneficial for the accurate evaluation of not only the primary lesions but also recurrences in postoperative 
Table 3: Qualitative evaluations and $\boldsymbol{\kappa}$ values for overall image quality, overall artifacts, and anatomic conspicuities of GRASP-VIBE and T1-TSE

\begin{tabular}{|c|c|c|c|c|c|c|c|}
\hline & \multicolumn{3}{|c|}{ GRASP-VIBE } & \multicolumn{3}{|c|}{ TI-TSE } & \multirow[b]{2}{*}{$P$ Value ${ }^{b}$} \\
\hline & Scores (mean) & $\kappa$ & $P$ Value $^{\mathrm{a}}$ & Scores (mean) & $\kappa$ & $P$ Value ${ }^{\mathrm{a}}$ & \\
\hline \multicolumn{8}{|l|}{ Overall image quality } \\
\hline Rater A & $4.74(S D, 0.49)$ & 0.29 & .003 & 3.81 (SD, 0.68) & 0.594 & $<.001$ & \\
\hline Rater B & $4.62(S D, 0.53)$ & & & $3.51(\mathrm{SD}, 0.91)$ & & & \\
\hline Average of two raters & $4.68(S D, 0.41)$ & & & $3.66(S D, 0.73)$ & & & $<.001$ \\
\hline Overall artifacts & & 0.21 & .041 & & 0.542 & $<.001$ & \\
\hline Rater A & $4.26(S D, 0.68)$ & & & $3.60(S D, 0.60)$ & & & \\
\hline Rater B & $4.68(S D, 0.51)$ & & & $3.55(S D, 0.97)$ & & & \\
\hline Average of two raters & $4.47(S D, 0.48)$ & & & $3.58(S D, 0.71)$ & & & $<.001$ \\
\hline Oropharyngeal mucosal conspicuity & & 0.80 & $<.001$ & & 0.5 & $<.001$ & \\
\hline Rater A & $4.87(S D, 0.39)$ & & & $4.15(S D, 0.74)$ & & & \\
\hline Rater B & $4.83(S D, 0.47)$ & & & $4.04(S D, 1.06)$ & & & \\
\hline Average of two raters & $4.85(S D, 0.41)$ & & & $4.11(\mathrm{SD}, 0.79)$ & & & $<.001$ \\
\hline Hypopharyngeal mucosal conspicuity & & 0.30 & .016 & & 0.422 & .002 & \\
\hline Rater A & 4.89 (SD, 0.32) & & & $3.58(S D, 0.84)$ & & & \\
\hline Rater B & 4.79 (SD, 0.49) & & & 3.57 (SD, 1.05) & & & \\
\hline Average of two raters & $4.84(S D, 0.34)$ & & & $3.58(\mathrm{SD}, 0.81)$ & & & $<.001$ \\
\hline Cervical lymph node conspicuity & & 0.18 & .072 & & 0.34 & .001 & \\
\hline Rater A & $4.64(S D, 0.48)$ & & & $4.34(S D, 0.65)$ & & & \\
\hline Rater B & $4.94(S D, 0.30)$ & & & $3.81(\mathrm{SD}, 0.83)$ & & & \\
\hline Average of two raters & $4.79(S D, 0.32)$ & & & $4.08(S D, 0.64)$ & & & $<.001$ \\
\hline
\end{tabular}

Qualitative assessments of GRASP-VIBE and T1-TSE

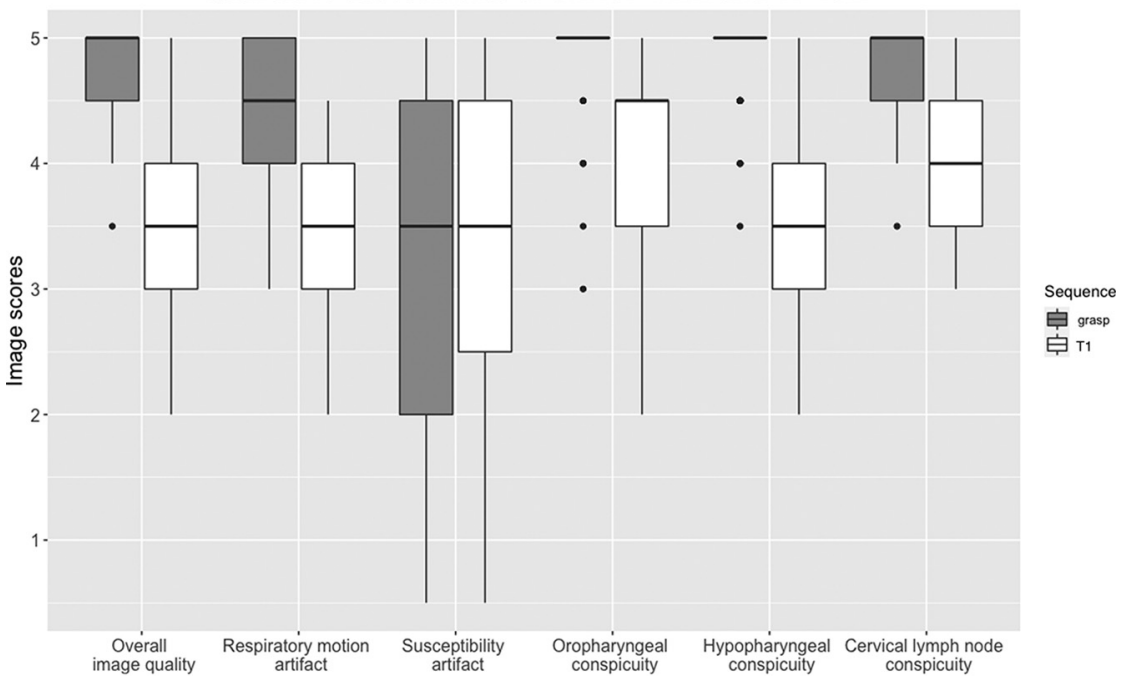

FIG 4. Boxplots of qualitative assessments for GRASP-VIBE and TT-TSE.

The potential benefit of GRASPVIBE over T1-TSE lies in its 3D reconstruction capability for coronal and sagittal images. While the protocol of the current study involved only GRASP-VIBE for high temporal resolution, GRASP-VIBE can also be acquired with high spatial resolution. Finally, the functional application of GRASP-VIBE in evaluating tumor angiogenesis has also been previously investigated in lung cancer, ${ }^{17}$ rectal cancer, ${ }^{18}$ and parotid neoplasms, ${ }^{19}$ proving its $4 \mathrm{D}$ imaging characteristics.

The GRASP-VIBE sequence has a few weaknesses compared with T1TSE. GRASP-VIBE is more prone to susceptibility artifacts than T1-TSE because the gradient recalled-echo sequence has no $180^{\circ}$ refocusing pulse, which limits correction for large and fixed magnetic field inhomogeneities

follow-up MR imaging, in which anatomic complexities become more challenging in the head and neck region.

Our findings are consistent with those in a previous similar study by Wu et al, ${ }^{11}$ who compared radial VIBE with conventional T1-TSE and found that radial VIBE was more motion-robust. The motion-robust benefit of radial VIBE has been documented in other anatomic regions as well, such as the abdomen ${ }^{16}$ and prostate. ${ }^{9}$ The added value of the present study is the application of a novel GRASP-VIBE technique and its direct intraindividual comparison with the conventional T1-TSE for head and neck MR imaging in routine clinical settings. induced by metallic implants. ${ }^{20}$ In head and neck MR imaging, significant susceptibility artifacts are often seen in the oral cavity due to dental amalgams; we found that fat suppression in GRASPVIBE was weaker in submental regions due to local signal loss caused by severe susceptibility change and local magnetic inhomogeneity in the oral cavity. However, the fat-saturation techniques for GRASP-VIBE and T1-TSE were different-chemical shift selective and Dixon, respectively-which might have contributed to the difference in fat-suppression effects. Furthermore, the radial sampling of $k$-space in GRASP-VIBE inherently leads to undersampling of peripheral $k$-spaces, resulting in the edge smoothing of 


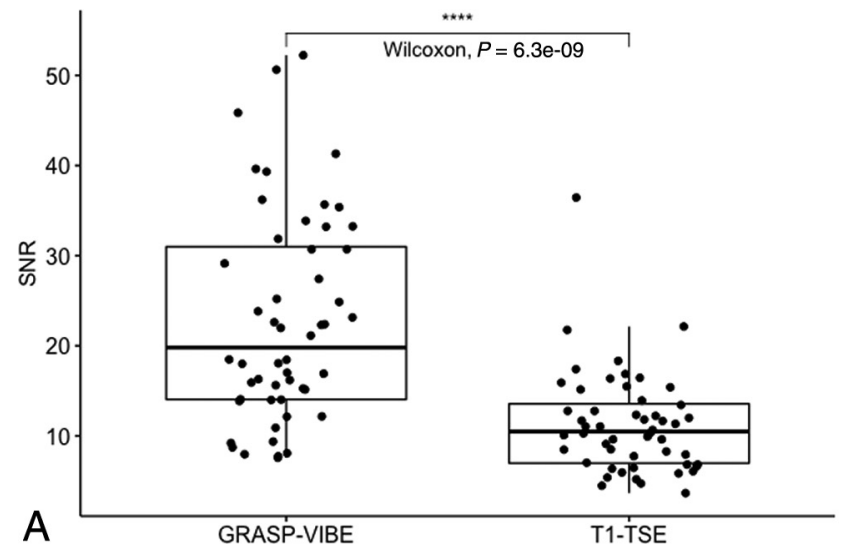

FIG 5. Boxplots of SNR (A) and CNR (B) of GRASP-VIBE and TI-TSE.

images. $^{21}$ Nonetheless, reduction of significant motion artifacts would compensate for such smoothing around the borders of structures.

This study had several limitations that need to be addressed. First, the 2 raters were not blinded to the names of the sequences during image analysis, possibly causing selection bias. However, the 2 sequences demonstrated obvious differences in image texture, and blinding would have had a limited role. Additionally, there was an interval of approximately 45 minutes between the GRASP-VIBE and T1-TSE sequences, which might have affected the extent and degree of tissue contrast enhancement. Finally, interobserver agreement was variable across qualitative assessment categories, possibly due to the differences in the experience of the raters ( 2 versus 8 years in head and neck radiology).

\section{CONCLUSIONS}

In qualitative image assessment, GRASP-VIBE demonstrated better image quality, fewer artifacts, and better image conspicuities than conventional contrast-enhanced T1-TSE. GRASPVIBE also provided a significantly higher SNR than T1-TSE. Therefore, the results of the current study are consistent with our hypothesis that GRASP-VIBE is a promising alternative to T1-TSE for the evaluation of head and neck MR imaging.

\section{ACKNOWLEDGMENTS}

The authors sincerely appreciate the technical support and advice provided by Hyun-Soo Lee, PhD (MR research collaboration scientist, Siemens Healthineers).

\section{REFERENCES}

1. Barakos JA, Dillon WP, Chew WM. Orbit, skull base, and pharynx: contrast-enhanced fat suppression MR imaging. Radiology 1991;179:191-98 CrossRef Medline

2. Hirsch JA, Loevner LA, Yousem DM, et al. Gadolinium-enhanced fat-suppressed T1-weighted imaging of the head and neck: comparison of gradient and conventional SE sequences. J Comput Assist Tomogr 1998;22:771-76 CrossRef Medline

3. Ross MR, Schomer DF, Chappell $\mathrm{P}$, et al. MR imaging of head and neck tumors: comparison of T1-weighted contrast-enhanced fatsuppressed images with conventional T2-weighted and fast spin-

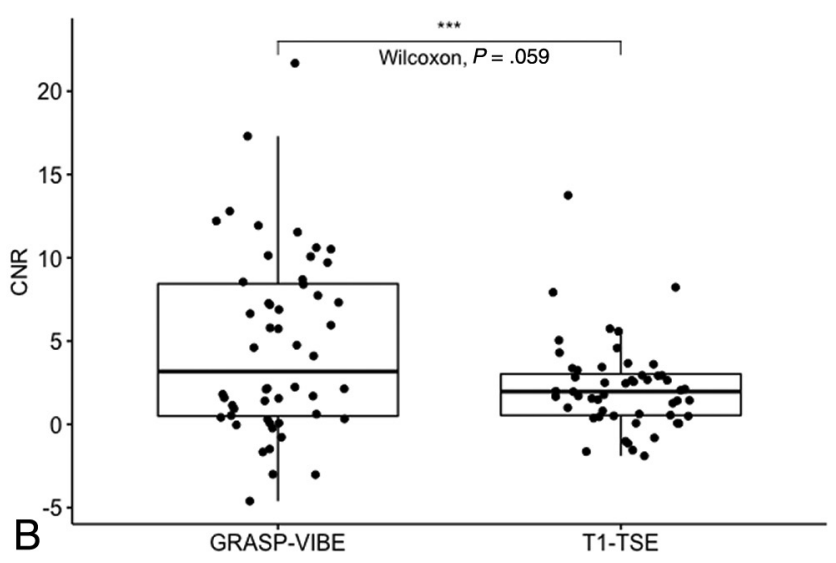

echo T2-weighted images. Am J Roentgenol 1994;163:173-78 CrossRef Medline

4. Kataoka M, Ueda H, Koyama T, et al. Contrast-enhanced volumetric interpolated breath-hold examination compared with spin-echo T1weighted imaging of head and neck tumors. AJR Am J Roentgenol 2005;184:313-19 CrossRef Medline

5. Castelijns JA, Gerritsen GJ, Kaiser MC, et al. Invasion of laryngeal cartilage by cancer: comparison of CT and MR imaging. Radiology 1988;167:199-206 CrossRef Medline

6. Becker M, Zbären P, Laeng H, et al. Neoplastic invasion of the laryngeal cartilage: comparison of MR imaging and CT with histopathologic correlation. Radiology 1995;194:661-69 CrossRef Medline

7. Feng L, Grimm R, Block KT, et al. Golden-angle radial sparse parallel MRI: combination of compressed sensing, parallel imaging, and golden-angle radial sampling for fast and flexible dynamic volumetric MRI. Magn Reson Med 2014;72:707-17 CrossRef Medline

8. Chandarana H, Feng L, Block TK, et al. Free-breathing contrastenhanced multiphase MRI of the liver using a combination of compressed sensing, parallel imaging, and golden-angle radial sampling. Invest Radiol 2013;48:10-16 CrossRef Medline

9. Rosenkrantz AB, Geppert C, Grimm R, et al. Dynamic contrastenhanced MRI of the prostate with high spatiotemporal resolution using compressed sensing, parallel imaging, and continuous golden-angle radial sampling: preliminary experience. J Magn Reson Imaging 2015;41:1365-73 CrossRef Medline

10. Heacock L, Gao Y, Heller SL, et al. Comparison of conventional DCE-MRI and a novel golden-angle radial multicoil compressed sensing method for the evaluation of breast lesion conspicuity. $J$ Magn Reson Imaging 2017;45:1746-52 CrossRef Medline

11. Wu X, Raz E, Block TK, et al. Contrast-enhanced radial 3D fatsuppressed $\mathrm{T} 1$-weighted gradient-recalled echo sequence versus conventional fat-suppressed contrast-enhanced T1-weighted studies of the head and neck. Am J Roentgenol 2014;203:883-89 CrossRef Medline

12. Winkelmann S, Schaeffter T, Koehler T, et al. An optimal radial profile order based on the golden ratio for time-resolved MRI. IEEE Trans Med Imaging 2007;26:68-76 CrossRef Medline

13. Ikeda $\mathrm{H}$, Ohno $\mathrm{Y}$, Murayama $\mathrm{K}$, et al. Compressed sensing and parallel imaging accelerated T2 FSE sequence for head and neck MR imaging: comparison of its utility in routine clinical practice. Eur J Radiol 2021;135:109501 CrossRef Medline

14. Vranic JE, Cross NM, Wang Y, et al. Compressed sensing-sensitivity encoding (CS-SENSe) accelerated brain imaging: reduced scan time without reduced image quality. AJNR Am J Neuroradiol 2019;40:9298 CrossRef Medline

15. Toledano-Massiah S, Sayadi A, De Boer R, et al. Accuracy of the compressed sensing accelerated 3D-FLAIR sequence for the detection of MS plaques at 3T. AJNR Am J Neuroradiol 2018;39:454-58 CrossRef Medline 
16. Seo N, Park SJ, Kim B, et al. Feasibility of free-breathing dynamic contrast-enhanced MRI of the abdomen: a comparison between CAIPIRINHA-VIBE, radial-VIBE with KWIC reconstruction and conventional VIBE. Br J Radiol 2016;89:1-7 CrossRef Medline

17. Chen $\mathrm{L}, \mathrm{Zeng} \mathrm{X}, \mathrm{Wu} \mathrm{Y}$, et al. A study of the correlation of perfusion parameters in high-resolution GRASP MRI with microvascular density in lung cancer. J Magn Reson Imaging 2019;49:1186-94 CrossRef Medline

18. Attenberger UI, Liu J, Riffel P, et al. Quantitative perfusion analysis of the rectum using golden-angle radial sparse parallel magnetic resonance imaging: initial experience and comparison to time- resolved angiography with interleaved stochastic trajectories. Invest Radiol 2017;52:715-24 CrossRef Medline

19. Mogen JL, Block KT, Bansal NK, et al. Dynamic contrast-enhanced MRI to differentiate parotid neoplasms using golden-angle radial sparse parallel imaging. AJNR Am J Neuroradiol 2019;40:1029-36 CrossRef Medline

20. Lee MJ, Kim S, Lee SA, et al. Overcoming artifacts from metallic orthopedic implants at high-field-strength $M R$ imaging and multidetector CT. Radiographics 2007;27:791-803 CrossRef Medline

21. Huang SY, Seethamraju RT, Patel P, et al. Body MR imaging: artifacts, $\mathbf{k}-$ space, and solutions. Radiographics 2015;35:1439-60 CrossRef Medline 